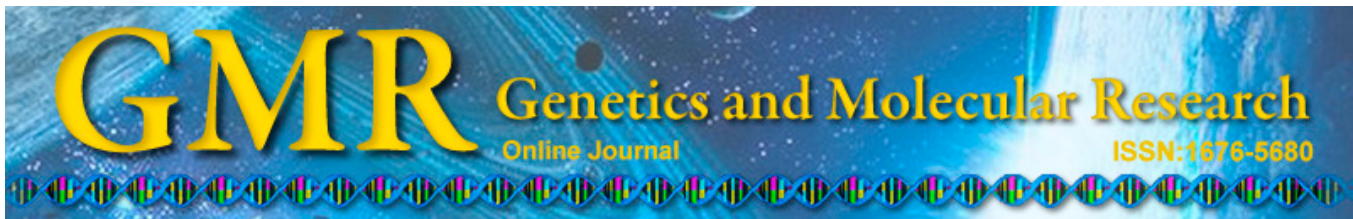

\title{
Natural variation of rice blast resistance gene Pi-d2
}

\author{
J.B. Li ${ }^{1 *}$, Y.D. Sun ${ }^{2,3,4 *}$, H. Liu ${ }^{5,6}$, Y.Y. Wang ${ }^{2,3,4}$, Y.L. Jia ${ }^{7}$ and M.H. Xü ${ }^{2,3,4}$ \\ ${ }^{1}$ Agricultural Environment and Resources Research Institute, \\ Yunnan Academy of Agricultural Sciences, Kunming, Yunnan Province, China \\ ${ }^{2}$ The Key Laboratory of Biotechnology Research of Yunnan Province, \\ Yunnan Academy of Agricultural Sciences, Kunming, Yunnan Province, China \\ ${ }^{3}$ Key Lab of Southwestern Crop Gene Resources and Germplasm Innovation \\ of Ministry of Agriculture, Yunnan Academy of Agricultural Sciences, \\ Kunming, Yunnan Province, China \\ ${ }^{4}$ Institute of Biotechnology and Genetic Resource, \\ Yunnan Academy of Agricultural Sciences, Kunming, Yunnan Province, China \\ ${ }^{5}$ Kunming Institute of Zoology, Chinese Academy of Sciences, Kunming, \\ Yunnan Province, China \\ ${ }^{6}$ Graduate University of Chinese Academy of Sciences, Beijing, China \\ ${ }^{7}$ United States Department of Agriculture-Agricultural Research Service, \\ Dale Bumpers National Rice Research Center, Stuttgart, Arkansas, USA \\ *These authors contributed equally to this study. \\ Corresponding authors: M. Xu / Y. Jia \\ E-mail: xuminhui@sohu.com / Yulin.Jia@ars.usda.gov
}

Genet. Mol. Res. 14 (1): 1235-1249 (2015)

Received November 25, 2013

Accepted March 20, 2014

Published February 13, 2015

DOI http://dx.doi.org/10.4238/2015.February.13.2

ABSTRACT. Studying natural variation in rice resistance genes of cultivated and wild rice relatives can predict resistance stability to rice blast fungus. In the present study, the protein coding regions of the rice $R$ gene Pi-d2 in 35 rice accessions, including Oryza sativa L. subsp. indica Kato (Aus), indica (IND), temperate japonica (TEJ), tropical japonica (TRJ), aromatic (ARO); subgroups of Oryza sativa; 6 accessions of wild rice varieties; $O$. nivara; and $O$. rufipogon were analyzed. A total of 13 
nucleotide differences were found in the open reading frames (ORFs) of $P i-d 2$. Translation of these ORFs revealed 9 variants; 3 were novel $P i-d 2$ variants. Variants $\mathrm{H} 2$ and $\mathrm{H} 5$ were identified in accessions of cultivated rice and $O$. nivara, $\mathrm{H} 1, \mathrm{H} 3, \mathrm{H} 4, \mathrm{H} 6$, and $\mathrm{H} 8$ were only identified in cultivated rice. $\mathrm{H} 2$ and $\mathrm{H} 5$ were the common types of IND and $O$. nivara, H8 was the common type of TRJ and AUS, H6 was the specific type of AUS, and $\mathrm{H} 3$ was the specific type of ARO. H7 and H9 were specific haplotypes of $O$. nivara and $O$. rufipogon, respectively. These findings demonstrate that $P i-d 2$ variants are useful indicators for each subgroup, and $P i-d 2$ is an ancient gene that predates speciation of rice subgroups.

Key words: Resistance gene; Natural variation; Blast disease; Oryza sativa; Pi-d2

\section{INTRODUCTION}

Rice blast, caused by the filamentous ascomycete fungus (Magnaporthe oryzae), is one of the most damaging diseases to rice worldwide. Genetic resistance has been defined as major and minor gene-mediated resistance. Major gene-mediated resistance $(R)$ is effective in recognizing strains of the fungus that contain the corresponding avirulence $(A V R)$ gene, and major $R$ gene is complete and powerful; however, such resistance can be easily overcome by $M$. oryzae through mutation (Silue et al., 1992; Xing et al., 2013). Mutations of the AVR gene, AVR-Pital, in historical and contemporary field isolates have been well documented in defeating major gene-mediated resistance (Zhou et al., 2007; Dai et al., 2010; Xing et al., 2013). Blast disease is currently managed by the use of resistant cultivars that carry both the major and minor $R$ genes and the application of fungicides integrated into cultural practices. Among them, the use of resistant cultivars is the most economical and environmentally benign method of reducing crop loss due to blast. Searching and stacking $R$ genes with different resistance specificities has been a priority for rice breeders and pathologists worldwide (Hao et al., 2009). To date, over hundreds of major and minor rice blast $R$ genes have been identified, 19 of which (including Pi-d2) have been cloned (Ballini et al., 2008; Sharma et al., 2012).

Sequence analysis of natural alleles of cloned rice blast $R$ genes in the rice germplasm is useful for understanding the evolution of $R$ genes and predicting their origins (Okuyama et al., 2011). To date, extensive evolutionary analyses of $P i$-ta, $P i k$, and $P i 2 / 9$ have been well documented (Wang et al., 2008; Lee et al., 2009, 2011). As a result, DNA markers were also developed from portions of genes for marker-assisted selection (MAS), such as Pi-ta (Jia 2003; Jia et al., 2002, 2003, 2004), Pi-b (Fjellstrom et al., 2004), and Pi-km (Costanzo and Jia, 2010). These DNA markers have been effectively used for genetic studies and MAS worldwide (Hittalmani et al., 2000).

The $P i-d 2$ gene found in the indica cultivar Digu confers resistance to M. oryzae strain ZB15 (Chen et al., 2010a,b). Digu-containing Pi-d2 is particularly important for breeding resistance against rice blast disease in Southern China (Chen et al., 2004). Digu A, a carrier of $P i-d 2$ was used as a cytoplast male sterile donor in 1995 (Lei et al., 1998), which resulted in the release of Fuyi A, thus forming the basis for the release of 16 hybrid rice cultivars in the 1990s. Ever since their release, they have been grown in Fujian, Hubei, Sichuan, Guangxi, and Hunan Provinces in China, and the estimated cropland use for these cultivars is 130,000 ha annually. Meanwhile, Fuyi A has been used as an important cytoplasmic male sterile parent 
for improving rice blast resistance in 3 lines of hybrids in Southern China (Lei et al., 2004).

$P i-d 2$, a single copy gene within the rice genome, was cloned from a region near the centromere of chromosome 6 and encodes a putative serine-threonine receptor-like kinase (RLK) membrane-spanning protein with 825 amino acids (Chen et al., 2006). Because of its novel extracellular domain, $P i-d 2$ represents a new class of plant $R$ genes (Chen et al., 2006). A single amino acid at position 441 of the $\mathrm{Pi}-\mathrm{d} 2$ protein distinguishes resistant and susceptible alleles. The amino acid methionine (M) at 441 was found in the susceptible pi-d2 allele, and isoleucine (I) at 441 was found in the resistant allele.

The objectives of the present study were to determine variation in DNA and protein sequences of Pi-d 2 and analyze the natural variation of $P i-d 2$ in 41 cultivated and wild rice relatives worldwide.

\section{MATERIAL AND METHODS}

\section{Plant materials}

A total of 50 accessions of cultivated and wild rice were previously resequenced (Xu et al., 2011). From these 50 accessions, a total of 41 accessions were selected, which included 35 Asian cultivated rice and 6 wild rice accessions based on the quality of DNA sequences and their representations of the rice blast $R$ genes (Table 1). The 35 Asian cultivated rice accessions included 10 tropical japonica (TRJ), 8 temperate japonica (TEJ), 5 aromatic (ARO), 4 aus (AUS), and 8 indica (IND). Among them, IR36 was the only cultivar developed by the International Rice Research Institute (IRRI, Philippines). The remaining germplasms were landraces, including 6 wild rice accessions (including 1 accession of $O$. rufipogon) and 5 accessions of $O$. nivara. The sequenced variety Nipponbare was used as a reference. Germplasm accessions for pathogenicity assays were generously provided by Harold Bockelman of the United States Department of Agriculture (USDA), Agricultural Research Service (ARS), National Small Grains Collection, Aberdeen, Idaho, USA.

\begin{tabular}{|c|c|c|c|c|c|c|c|c|c|}
\hline Name of accessions & $\begin{array}{l}\text { Plant } \\
\text { identification }\end{array}$ & Status & Origin & $\begin{array}{l}\text { Variety } \\
\text { group }\end{array}$ & $\begin{array}{l}\text { Name of } \\
\text { accessions }\end{array}$ & $\begin{array}{l}\text { Plant } \\
\text { identification }\end{array}$ & Status & Origin & $\begin{array}{l}\text { Variety } \\
\text { group }\end{array}$ \\
\hline Mehr & IRGC 12883 & Landrace & Iran & AUS & Jambu & IRGC 17757 & Landrace & Indonesia & TRJ \\
\hline Kalamkati & IRGC 45975 & Landrace & India & AUS & AZUCENA & IRGC 328 & Landrace & Philippines & TRJ \\
\hline Jhona 349 & IRGC 6307 & Landrace & India & AUS & NPE 844 & IRGC 38698 & Landrace & Pakistan & TRJ \\
\hline DZ78 & IRGC 8555 & Landrace & Bangladesh & AUS & Arias & IRGC 43325 & Landrace & Indonesia & TRJ \\
\hline Leung Pratew & IRGC 27762 & Landrace & Thailand & IND & Gotak Gatik & IRGC 43397 & Landrace & Indonesia & TRJ \\
\hline IR 36 & IRGC 30416 & Improve & Brazil & IND & Trembese & IRGC 43675 & Landrace & Indonesia & TRJ \\
\hline Popot 165 & IRGC 43545 & Landrace & Indonesia & IND & Canella De Ferro & IRGC 50448 & Elite & Brazil & TRJ \\
\hline Ai-Chiao-Hong & IRGC 51250 & Landrace & China & IND & Lemont & IRGC 66756 & Elite & USA & TRJ \\
\hline Guan-Yin-Tsan & IRGC 51300 & Landrace & China & IND & Davao & IRGC 8244 & Landrace & Philippines & TRJ \\
\hline Gie 57 & IRGC 8231 & Landrace & Vietnam & IND & Kitrana 508 & IRGC 12793 & Elite & Madagascar & ARO \\
\hline TD2 & IRGC 9148 & Elite & Thailand & IND & Bico Branco & IRGC 38994 & Elite & Brazil & ARO \\
\hline JC91 & IRGC 9177 & Elite & India & IND & JC101 & IRGC 9060 & Elite & India & ARO \\
\hline Ta Hung Ku & IRGC 1107 & Landrace & China & TEJ & JC111 & IRGC 9062 & Elite & India & ARO \\
\hline Haginomae Mochi & IRGC 2540 & Elite & Japan & TEJ & Firooz & RA 4952 & Landrace & Iran & ARO \\
\hline Darmali & IRGC 27630 & Landrace & Nepal & TEJ & $042 / 87 / 34$ & IRGC 105327 & Wild rice & India & Nivara \\
\hline Phudugey & IRGC 32399 & Landrace & Bhutan & TEJ & MV $89-80$ & IRGC 106105 & Wild rice & India & Nivara \\
\hline Norin 20 & IRGC 418 & Landrace & Japan & TEJ & L $89-12$ & IRGC 106154 & Wild rice & Laos & Nivara \\
\hline Chodongji & IRGC 55471 & Landrace & Korea & TEJ & HK 47 & IRGC 80470 & Wild rice & India & Nivara \\
\hline Mansaku & IRGC 8191 & Landrace & Japan & TEJ & CA $97-053$ & IRGC 89215 & Wild rice & Cambodia & Nivara \\
\hline Nipponbare & $\mathrm{NP}$ & Elite & Japan & TEJ & PADI PADIAN & IRGC 105958 & Wild rice & Indonesia & Rufipogon \\
\hline Maintmolotsy & IRGC 11010 & Elite & Madagascar & TRJ & & & & & \\
\hline
\end{tabular}

aThese germplasm accessions were described in Xu et al. (2012). 


\section{Sequence analysis}

The coding sequences of $P i-d 2$ in Digu (GenBank accession No. FJ915121.1) and Nipponbare (accession No. NC008399) were obtained from http://blast.ncbi.nlm.nih.gov. Pi$d 2^{\mathrm{Np}}(\mathrm{NC} 008399)$ and $P i-d 2^{\text {Digu }}($ FJ915121.1) were $99 \%$ identical and identified from nucleotide positions $18040176-18037639$ on rice chromosome 6 . The target fragments of the $P i-d 2$ alleles in the 41 rice accessions were obtained from the same location. Briefly, DNA sequences of chromosome 6 from the 41 rice accessions were opened by the Editplus v3.40 software (http:// www.editplus.com/files.html), and nucleotide sequences of each rice accession from 18040176 to 18037639 were extracted and saved to a new file. DNA sequences of Pi-d2 in 41 rice accessions were aligned using the DNASTAR v7.10 software (http://www.dnastar.com/). The diversity index was calculated as the frequency of haplotypes or protein types in the accession populations following Fontaine's method (Fontaine et al., 2004) by using the formula:

$$
\text { Diversity index }=\left(1-\sum_{i}^{n_{i}}=1 p_{i}{ }^{2}\right)
$$

where $\mathrm{p}_{i}$ is the frequency of the haplotype $i$ in a population. A phylogenetic tree was constructed using the DNASTAR v7.10 software by the ClustalW method. Tree view was constructed using the MEGA 4.0 software (Tamura et al., 2007).

\section{Nucleotide polymorphism analysis}

Nucleotide polymorphisms were calculated according to Rozas's methods by the DnaSP v5.10.01 software (Rozas et al., 2003). The aligned DNA sequences were imported into the DnaSP v5.10.01 program to calculate $S$ (number of polymorphic or segregating sites), $\pi$ (nucleotide diversity), $\theta$ (Theta from S, Theta-W), and $D$ (Tajima's $D$ ) (Tajima et al., 1989); then, we drew the sliding window of nucleotide diversity $(\pi)$.

\section{Positive selection analysis}

The positive selection analysis was performed using the Selection Server program downloaded from the website http://selecton.tau.ac.il/. Five calculation models were used to identify the positive selection sites under the query of Pid2 as follows: M8 (positive selection enabled, beta $+\mathrm{w}>=1$ ) (Yang et al., 2000), M8a (beta $+\mathrm{w}=1$, null model) (Swanson et al., 2003), M7 (beta, null model) (Yang et al., 2000), M5 (positive selection enabled, gamma) (Yang et al., 2000), and the mechanistic empirical combination (MEC) model (Doron-Faigenboim et al., 2006). The data were then imported into Microsoft Excel for statistical analysis and to draw the sliding window.

\section{Pathogenicity assay}

Pathogenicity assays were performed on 25 available rice accessions at the USDA ARS Dale Bumpers National Rice Research Center, Stuttgart, Arkansas, USA. M. oryzae isolates, one avirulent (AVR) race IB1 (ARB146), one virulent (VIR) race, IEIK (TM2) for rice cultivars carrying the rice blast $R$ gene $P i$-ta (Xing et al., 2013) were used for pathogenicity tests. There were 4 replicates for each germplasm. The reactions to rice blast were determined 
using a modified standard pathogenicity assay as previously described by Jia et al. (2003). Specifically, rice seedlings at the 3rd-4th leaf stages in a plastic bag were spray-inoculated with a rice blast spore suspension at $1-5 \times 10^{5}$ spores $/ \mathrm{mL}$. After inoculation, the plastic bags were sealed to maintain high humidity for $24 \mathrm{~h}$; subsequently, the plants were removed from the bags. Then, the plants were maintained in a greenhouse for an additional 6 days to allow for development of notable disease symptoms. The reactions were rated as $0-2$ for resistant and 3-5 for susceptible based on the visual number of lesions at the second youngest leaf.

\section{RESULTS}

\section{Natural variation in the $P i-d 2$ allele}

The length of the open reading frame (ORF) of $P i-d 2$ was found to be $2478 \mathrm{bp}$ in all rice accessions. Nucleotide variations were found at positions 962, 1323, 1997, 1998, 2172, and 2463 between the resistant $P i-d 2^{\text {Digu }}$ and susceptible $p i-d 2^{\mathrm{Np}}$, and a total of 13 nucleotide variations were found among the 41 rice accessions (Table 2). The variation frequencies at positions 1997, 1998, and 2172 in the 41 accessions were higher than those of the other regions. Nucleotides at these positions were different than those of $P i-d 2^{\text {Digu }}$ in most accessions. Among the 13 different positions, the same nucleotides at 495, 1998, and 2172 were detected in the cultivated rice and 2 species of wild rice, whereas 347 and 1997 were detected in the cultivated rice and $O$. nivara. However, the same nucleotide at position 1497 was detected only in $O$. rufipogon. These findings suggest that there are significant differences in the sequences of $P i-d 2$ between $O$. rufipogon and the cultivated rice.

\begin{tabular}{|c|c|c|c|c|c|c|c|c|c|c|c|c|c|}
\hline \multirow{2}{*}{$\begin{array}{l}\text { Variant } \\
\text { locus }\end{array}$} & \multirow{2}{*}{$\begin{array}{c}\text { No. of } \\
\text { sequenced } \\
\text { accessions }\end{array}$} & \multirow{2}{*}{$\begin{array}{c}\text { Bases in } \\
P i-d 2 \text { gene }\end{array}$} & \multirow{2}{*}{$\begin{array}{c}\text { Base in } \\
\text { Nipponbare }\end{array}$} & \multirow{2}{*}{$\begin{array}{l}\text { Substituted } \\
\text { bases }\end{array}$} & \multirow{2}{*}{$\begin{array}{c}\text { No. of } \\
\text { accessions }\end{array}$} & \multirow{2}{*}{$\begin{array}{c}\text { Frequency } \\
(\%)\end{array}$} & \multicolumn{7}{|c|}{ Number } \\
\hline & & & & & & & ARO & AUS & TEJ & TRJ & IND & Nivara & Rufipogon \\
\hline 20 & 41 & $\mathrm{G}$ & - & $\mathrm{A}$ & 1 & 2.4 & 0 & 0 & 0 & 0 & 0 & 1 & 0 \\
\hline 66 & 41 & $\mathrm{~A}$ & - & $\mathrm{T}$ & 5 & 12.2 & 5 & 0 & 0 & 0 & 0 & 0 & 0 \\
\hline 347 & 41 & $\mathrm{~T}$ & - & A & 3 & 7.3 & 0 & 0 & 0 & 0 & 2 & 1 & 0 \\
\hline 495 & 41 & G & - & A & 10 & 24.4 & 0 & 0 & 1 & 2 & 3 & 2 & 1 \\
\hline 962 & 41 & $\mathrm{C}$ & $\mathrm{T}$ & $\mathrm{T}$ & 15 & 36.6 & 0 & 2 & 8 & 5 & 0 & 0 & 0 \\
\hline 1107 & 41 & $\mathrm{G}$ & - & $\mathrm{A}$ & 5 & 12.2 & 5 & 0 & 0 & 0 & 0 & 0 & 0 \\
\hline 1323 & 41 & $\mathrm{~A}$ & G & $\mathrm{G}$ & 15 & 36.6 & 0 & 2 & 8 & 5 & 0 & 0 & 0 \\
\hline 1497 & 41 & $\mathrm{G}$ & - & A & 1 & 2.4 & 0 & 0 & 0 & 0 & 0 & 0 & 1 \\
\hline 1719 & 41 & $\mathrm{C}$ & - & $\mathrm{T}$ & 1 & 2.4 & 0 & 0 & 0 & 1 & 0 & 0 & 0 \\
\hline 1997 & 41 & A & G & G & 41 & 100 & 5 & 4 & 8 & 10 & 8 & 5 & 0 \\
\hline 1998 & 41 & $\mathrm{~T}$ & $\mathrm{C}$ & $\mathrm{C}$ & 33 & 80.5 & 5 & 4 & 8 & 10 & 3 & 2 & 1 \\
\hline 2172 & 41 & $\mathrm{~T}$ & $\mathrm{C}$ & $\mathrm{C}$ & 33 & 80.5 & 5 & 4 & 8 & 10 & 3 & 2 & 1 \\
\hline 2463 & 41 & $\mathrm{C}$ & $\mathrm{T}$ & $\mathrm{T}$ & 15 & 36.6 & 0 & 2 & 8 & 5 & 0 & 0 & 0 \\
\hline
\end{tabular}

${ }^{\mathrm{b}} \mathrm{GenBank}$ accession numbers for these germplasm were KB738450-738490 that will be provided upon acceptance of the manuscript.

Nucleotide variation at position 20 was only detected in one accession of $O$. nivara, and the same nucleotide at positions $66,962,1107,1323$, and 1719 was detected only in the cultivated rice. There were 1-7 nucleotide substitutions among the 35 cultivated rice accessions (Table 3). Among them, the same nucleotide was identified only at position 1719 in one accession of TRJ, whereas the same nucleotide at position 66 and 1107 was only identified in one 
accession of ARO (Table 2). These results suggest that DNA sequence variation for $P i-d 2$ can be used to distinguish between cultivated and wild rice, and among cultivated rice accessions.

Table 3. Haplotypes of $P i-d 2$ ORF in different accessions.

\begin{tabular}{|c|c|c|c|c|c|c|c|c|c|c|c|c|c|c|c|}
\hline \multirow[t]{2}{*}{ Haplotypes } & \multicolumn{13}{|c|}{ Varied locus in ORF of $P i-d 2$} & \multirow{2}{*}{$\begin{array}{c}\text { No. of } \\
\text { variant } \\
\text { bases }\end{array}$} & \multirow[t]{2}{*}{ Accessions } \\
\hline & 20 & 66 & 347 & 495 & 962 & 1107 & 1323 & 1497 & 1719 & 1997 & 1998 & 2172 & 2463 & & \\
\hline$P i-d 2^{\text {Digu }}$ & G & A & $\mathrm{T}$ & G & $\mathrm{C}$ & G & A & G & $\mathrm{C}$ & A & $\mathrm{T}$ & $\mathrm{T}$ & $\mathrm{C}$ & & Digu \\
\hline$P i-d 2^{\mathrm{Np}}$ & $-b$ & - & - & - & $\mathrm{T}$ & - & G & - & - & G & $\mathrm{C}$ & $\mathrm{C}$ & $\mathrm{T}$ & 6 & Nipponbare \\
\hline H1 & - & - & - & - & $\mathrm{T}$ & - & G & - & - & G & $\mathrm{C}$ & $\mathrm{C}$ & $\mathrm{T}$ & 6 & $\begin{array}{l}\text { IRGC12883, IRGC8555, IRGC1107, } \\
\text { IRGC2540, IRGC27630, IRGC32399, } \\
\text { IRGC418, IRGC55471, IRGC8191, } \\
\text { NP, IRGC11010, IRGC38698, } \\
\text { IRGC66756, IRGC8244 }\end{array}$ \\
\hline $\mathrm{H} 2$ & - & - & - & - & - & - & - & - & - & G & - & - & - & 1 & $\begin{array}{l}\text { IRGC27762, IRGC43545, IRGC51300, } \\
\text { IRGC9148, IRGC9177, IRGC105327, } \\
\text { IRGC106154, IRGC89215 }\end{array}$ \\
\hline $\mathrm{H} 3$ & - & $\mathrm{T}$ & - & - & - & A & - & - & - & G & $\mathrm{C}$ & $\mathrm{C}$ & - & 5 & $\begin{array}{l}\text { IRGC12793, IRGC38994, IRGC9060, } \\
\text { IRGC9062, RA } 4952\end{array}$ \\
\hline $\mathrm{H} 4$ & - & - & - & A & - & - & - & - & - & G & $\mathrm{C}$ & $\mathrm{C}$ & - & 4 & $\begin{array}{l}\text { IRGC51250, IRGC17757, IRGC43325, } \\
\text { IRGC43675, IRGC50448 }\end{array}$ \\
\hline H5 & - & - & A & A & - & - & - & - & - & $\mathrm{G}$ & $\mathrm{C}$ & C & - & 5 & IRGC30416, IRGC8231, IRGC80470 \\
\hline H6 & - & - & - & - & $\mathrm{T}$ & - & G & - & $\mathrm{T}$ & G & $\mathrm{C}$ & $\mathrm{C}$ & $\mathrm{T}$ & 7 & IRGC328 \\
\hline H7 & A & - & - & $\mathrm{A}$ & - & - & - & - & - & G & $\mathrm{C}$ & $\mathrm{C}$ & - & 5 & IRGC106105 \\
\hline H8 & - & - & - & - & - & - & - & - & - & G & $\mathrm{C}$ & $\mathrm{C}$ & - & 3 & IRGC43397, IRGC45975, IRGC6307 \\
\hline H9 & - & - & - & A & - & - & - & A & - & $\mathrm{G}$ & $\mathrm{C}$ & $\mathrm{C}$ & - & 5 & IRGC105958 \\
\hline
\end{tabular}

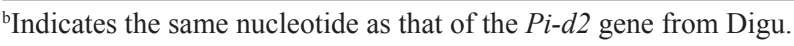

The nucleotide diversity of $P i-d 2$ ORFs was examined among the 41 sequence assemblies using the DnaSP program (Table 4). A total of 10 and 6 polymorphic sites were observed in the cultivated and wild rice accessions, respectively (Table 4). In the cultivated rice, the value of the nucleotide diversity $(\pi)$ was 0.00122 , which was higher than that of the wild rice group ( $\pi=0.00113)$, suggesting higher diversity in the cultivated rice group (Table 4). Furthermore, the wild rice $O$. nivara showed much higher diversity than that of the cultivated rice subgroups (i.e., AUS, TEJ, TRJ, IND, and ARO), suggesting a slightly higher diversity in $O$. nivara (Table 4). Interestingly, the nucleotide diversity was extremely low for ORFs in TEJ and ARO; the values of $S, \pi$, and $\theta$ were zero (Table 4 ). In $O$. sativa, TRJ had the lowest diversity $(\pi=0.00095$ and $\theta=0.00071)$ in the ORFs (Table 4 ), whereas similar values of $\pi$ and $\theta$ for ORFs were found in AUS and IND. These results suggest that the $P i-d 2$ alleles of $O . n i$ vara were subjected to positive selection. The $O$. sativa subgroups with low values of $\pi$ and $\theta$ (Table 4) suggest that AUS, TRJ, TEJ, IND, and ARO had been subjected to purified selection.

In order to examine the evolutionary dynamics of the $P i-d 2$ alleles in the cultivated and wild rice groups, we evaluated neutral selection with Tajima's $D$ test regardless of demographic consideration. Whereas, Tajima's $D$ in all rice accessions was not statistically significant, a positive Tajima's $D(0.40276)$ was observed among all accessions (Table 4), suggesting that the $P i-d 2$ alleles may have experienced purified selection. The Selection Server was used to identify the positive-selection sites in the $P i-d 2$ alleles, and we calculated the value of $\mathrm{Ka} /$ $\mathrm{Ks}(\mathrm{Ka}=$ the rate of nonsynonymous substitutions, $\mathrm{Ks}=$ the rate of synonymous substitutions) for each amino acid of the deduced Pi-d2-like protein sequences from the 41 accessions. Five models, including 3 positive-selection models, M8 (Yang et al., 2000), M5 (Yang et al., 2000), and MEC; and 2 null models, M7 (Yang et al., 2000), and M8a (Swanson et al., 2003), were 
tested using the Selection Server program (http://selecton.tau.ac.il/). In comparison to the null model M8a, the likelihood ratio test (LRT) of the M8 model was not significant in all rice groups (Tables 5 and 6 ). The results of 3 calculated positive-selection models were inconsistent (Tables 5 and 6). The sliding windows were drawn to show the distribution of the $\mathrm{Ka} / \mathrm{Ks}$ values across all 825 amino acids under the M5, M8, and MEC models (Figure 1). The results showed that the $\mathrm{Ka} / \mathrm{Ks}$ values of most sites $(\sim 90 \%)$ were $<1$, suggesting that these sites were potentially subjected to purifying selection. Few positive-selection sites $(\mathrm{Ka} / \mathrm{Ks}>1)$ with statistically significant results were found in the ORFs.

\begin{tabular}{|c|c|c|c|c|c|c|c|}
\hline Group & Subgroup & Component & Location (nt) & $S$ & $\pi$ & $\theta$ & Tajima's $D^{\mathrm{j}}$ \\
\hline All & & Total & $1-2,478$ & 12 & 0.00128 & 0.00113 & 0.40276 \\
\hline \multirow{6}{*}{ Cultivated rice } & & Total & $1-2,478$ & 10 & 0.00122 & 0.00098 & 0.74480 \\
\hline & AUS & Total & $1-2,478$ & 3 & 0.00081 & 0.00066 & 2.01187 \\
\hline & TEJ & Total & $1-2,478$ & 0 & 0 & 0 & 0 \\
\hline & TRJ & Total & $1-2,478$ & 5 & 0.00095 & 0.00071 & 1.43514 \\
\hline & IND & Total & $1-2,478$ & 4 & 0.00082 & 0.00062 & 1.39420 \\
\hline & ARO & Total & $1-2,478$ & 0 & 0 & 0 & 0 \\
\hline \multirow[t]{2}{*}{ Wild rice } & & Total & $1-2,478$ & 6 & 0.00113 & 0.00106 & 0.37522 \\
\hline & O. nivara & Total & $1-2,478$ & 5 & 0.00105 & 0.00097 & 0.56199 \\
\hline
\end{tabular}

${ }^{\mathrm{f}} \mathrm{S}$ number of polymorphic or segregating sites; $\pi$ nucleotide diversity; $\theta$ Watterson's nucleotide diversity estimator based on silent site. jTajima's $D$ statistic (Tajima et al., 1989) based on the differences between the number of segregating sites and the average number of nucleotide differences.

\begin{tabular}{|c|c|c|c|}
\hline Group & Model & Significance level & Total positive sites \\
\hline \multirow{3}{*}{ All } & M8 & $\mathrm{NON}^{\mathrm{g}}$ & 0 \\
\hline & M5 & - & 0 \\
\hline & MEC & $\mathrm{AIC}>\mathrm{M} 8 \mathrm{a}^{\mathrm{h}}$ & 4 \\
\hline \multirow[t]{3}{*}{ Cultivated rice } & M8 & NON & 0 \\
\hline & M5 & - & 0 \\
\hline & MEC & $\mathrm{AIC}>\mathrm{M} 8 \mathrm{a}$ & 4 \\
\hline \multirow[t]{3}{*}{ Wild rice } & M8 & NON & 0 \\
\hline & M5 & - & 0 \\
\hline & MEC & $\mathrm{AIC}<\mathrm{M} 8 \mathrm{a}$ & 2 \\
\hline
\end{tabular}

The positive-selection sites with a statistical significance (the lower bound of confidence interval $>1$ ) under Bayesian test was used for calculation here. IIndicates likelihood ratio test between the two models (M8 and M8a) shows nonsignificant. "'If the score of AIC under MEC model is less than the value of M8a, it is statistically significant.

\section{Distribution of $\mathrm{Pi}-\mathrm{d} 2$ haplotypes in rice accession}

Half of the accessions in AUS were $\mathrm{H} 1$ and $\mathrm{H} 8 ; 62.5,25.0$, and $12.5 \%$ of accessions in IND were $\mathrm{H} 2, \mathrm{H} 5$, and $\mathrm{H} 4$, respectively. All of the accessions of ARO and TEJ were H3, and $40.0 \%$ of the accessions in TRJ were $\mathrm{H} 1$ and H4. Four haplotypes were detected in 10 accessions of TRJ. The diversity index of haplotypes was calculated as 0.66 ; TRJ possessed the highest diversity. Similarly, the diversity indices were $0.56,0.53$, and 0.50 , for O. nivara, IND, and AUS, respectively. The lowest diversity index was 0.00 , which was found in TEJ, ARO, and $O$. rufipogon (Table 7). In summary, the diversity indices of $P i-d 2$ can be ordered as follows: TRJ $>$ O. nivara $>$ IND $>$ AUS $>$ O. rufipogon, ARO, and TEJ. 
Table 6. Nucleotide polymorphism of the $P i-d 2$ alleles in different rice subgroups ${ }^{\mathrm{g}}$.

\begin{tabular}{|c|c|c|c|c|}
\hline Group & Subgroup & Model & Significance level & Total positive sites \\
\hline \multirow[t]{15}{*}{ Cultivated rice } & \multirow[t]{3}{*}{ AUS } & M8 & NON"g & 2 \\
\hline & & M5 & - & 2 \\
\hline & & MEC & $\mathrm{AIC}>\mathrm{M} 8 \mathrm{a}^{\mathrm{h}}$ & 0 \\
\hline & \multirow[t]{3}{*}{ TEJ } & M8 & NON & 0 \\
\hline & & M5 & - & 0 \\
\hline & & MEC & $\mathrm{AIC}>\mathrm{M} 8 \mathrm{a}$ & 825 \\
\hline & \multirow[t]{3}{*}{ TRJ } & M8 & non & 0 \\
\hline & & M5 & - & 0 \\
\hline & & MEC & $\mathrm{AIC}<\mathrm{M} 8 \mathrm{a}$ & 2 \\
\hline & \multirow[t]{3}{*}{ IND } & M8 & NON & 0 \\
\hline & & M5 & - & 0 \\
\hline & & MEC & $\mathrm{AIC}<\mathrm{M} 8 \mathrm{a}$ & 1 \\
\hline & \multirow[t]{3}{*}{ ARO } & M8 & NON & 0 \\
\hline & & M5 & - & 0 \\
\hline & & MEC & $\mathrm{AIC}>\mathrm{M} 8 \mathrm{a}$ & 825 \\
\hline \multirow[t]{3}{*}{ Wild rice } & \multirow[t]{3}{*}{ O. nivara } & M8 & NON & 0 \\
\hline & & M5 & - & 0 \\
\hline & & MEC & $\mathrm{AIC}<\mathrm{M} 8 \mathrm{a}$ & 2 \\
\hline
\end{tabular}

The positive-selection sites with a statistical significance (the lower bound of confidence interval $>1$ ) under Bayesian test was used for calculation here. ${ }^{\mathrm{g}}$ Indicates likelihood ratio test between the two models (M8 and M8a). hIf the score of AIC under MEC model is less than the value of M8a, it is statistically significant.

\section{Functional amino acid of the Pi-d2 protein}

Alignment of DNA sequence assemblies revealed $9 P i-d 2$ haplotypes (H) (Table 3). A previous study by others demonstrated that the reference cultivar Nipponbare, belonging to H1, was the susceptible $p i-d 2$ haplotype (Chen et al., 2006), whereas the remaining $8 p i-d 2$ haplotypes were different than those of $P i-d 2^{\text {Digu }}$ and $P i-d 2^{\mathrm{Np}}$. H1 was found in 14 rice accessions (Table 7), with the highest frequency of haplotype variation (34.1\%) among the 9 haplotypes (Table 7). There were 6 nucleotide substitutions from $P i-d 2^{\mathrm{Np}}$ compared to those for $P i-d 2^{\text {Digu }}$. The second highest frequency in nucleotide variation $(19.5 \%)$ was $\mathrm{H} 2$, which was found in 8 rice accessions. There was only one nucleotide variation when $P i-d 2^{\mathrm{Np}}$ was compared to $P i-d 2^{\text {Digu }}$. The third highest frequency of nucleotide variation $(12.2 \%)$ was found for $\mathrm{H} 3$ and $\mathrm{H} 4$ in 5 rice accessions. There were 5 and 4 nucleotide substitutions in $P i-d 2 \mathrm{H} 3$ and $P i-d 2 \mathrm{H} 4$, respectively, when compared to that of $P i-d 2^{\text {Digu }}$. The frequencies of nucleotide variation in the remaining haplotypes were $<10.0 \%$, with 3-5 nucleotide substitutions. Among the 9 haplotypes, $\mathrm{H} 2$ and $\mathrm{H} 5$ were found in rice cultivated and $O$. nivara; the $\mathrm{H} 1, \mathrm{H} 3, \mathrm{H} 4, \mathrm{H} 6$, and $\mathrm{H} 8$ haplotypes were found only in cultivated rice; $\mathrm{H} 2$ and $\mathrm{H} 5$ were the common types of IND and $O$. nivara. H6 was the specific type of TRJ, H3 was the specific type of ARO, H4 was the common type of IND and TRJ, and H8 was the common type of AUS and TRJ. H7 and H9 were specific haplotypes of $O$. nivara and $O$. rufipogon, respectively (Table 7). These results demonstrate that some rice cultivated and $O$. nivara share a few common $P i-d 2$ haplotypes, whereas the rice cultivated and $O$. nivara share no common haplotypes with $O$. rufipogon. These findings suggest that genetic differentiation of $P i-d 2$ between rice cultivated and $O$. nivara was lower in comparison to that of $O$. rufipogon.

Translation of the ORFs of $\mathrm{Pi}-\mathrm{d} 2$ haplotypes in 41 accessions revealed similar proteins with a putative serine-threonine RLK membrane-spanning protein of 825 amino acids. Pi-d2 also contained a predicted extracellular bulb-type mannose-specific lectin (B-lectin) binding domain, which has not been reported in other plant $\mathrm{R}$ proteins. The $\mathrm{N}$-terminus of $\mathrm{Pi}-\mathrm{d} 2$, amino 
acids 1-32, contains a hydrophobic region with a predicted transit peptide function (Wasano et al., 2003). Additionally, amino acids 337-418 are predicted to encode a weak PAN domain (smart e-02) that binds proteins or carbohydrates (Chen et al., 2006). The core of the PAN domain in the region of amino acids 337-403 is possibly connected to the formation of 3 disulphide bridges (Chen et al., 2006). Moreover, only the 441 st amino acid of Pi-d2 was reported as the functional locus for resistance or susceptibility. Among the amino acids of the Pi-d2 protein in the 41 accessions, there were 5 variant amino acids (i.e., R7H, F116Y, S321L, I441M, and H666R) when all Pi-d2 histidine $(\mathrm{H})$ were compared to those at the same position in Pi-d2 ${ }^{\text {Digu }}$. These variant amino acids were located in the functional domain of the N-terminal hydrophobic region, B-lectin of the extracellular domain, and serine-threonine kinase catalytic domain.
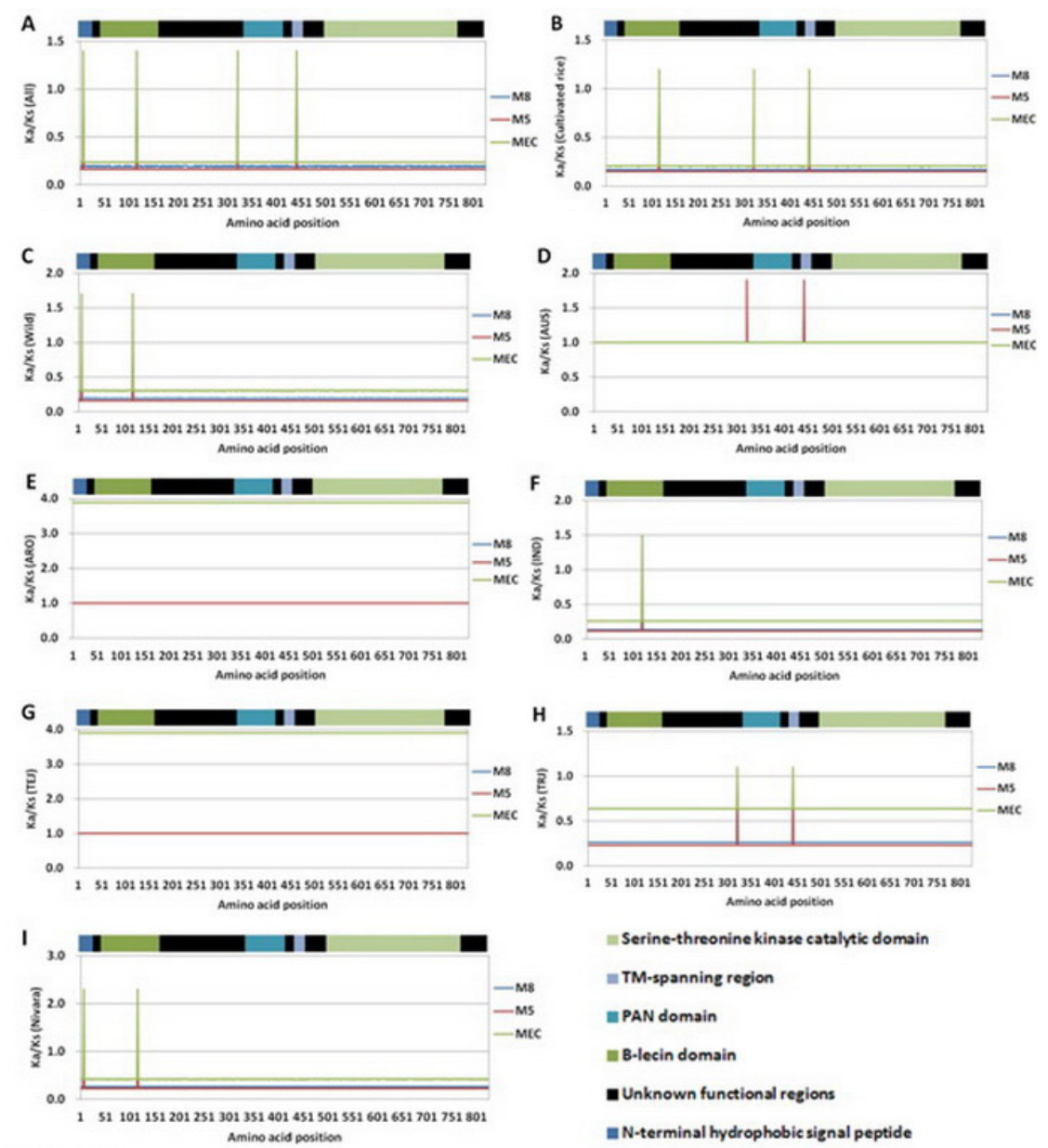

Figure 1. Sliding window of positive-selection sites of the $P i-d 2$ alleles under M8, M5, and MEC models. The Y-axis indicates the ratio of the rate of nonsynonmous substitution (Ka) to the rate of synonymous substitution $(\mathrm{Ks})$ $(\mathrm{Ka} / \mathrm{Ks})$; the $\mathrm{X}$-axis indicates the position of the Pi-d2 amino acids in the site. The graphic description of protein with predicted domains was above each sliding window. Different predicted domains were shaded with different color at the right end of figure. The sliding window showing the total rice accessions (A), cultivated rice (B), wild rice species $(\mathbf{C})$, AUS $(\mathbf{D})$, ARO $(\mathrm{E})$, IND $(\mathbf{F})$, TEJ $(\mathbf{G})$, TRJ $(\mathbf{H})$ and wild rice $O$. nivara $(\mathbf{I})$. 
Table 7. Distribution $P i-d 2$ haplotype in different rice groups.

\begin{tabular}{|c|c|c|c|c|c|c|c|c|c|}
\hline \multirow[t]{2}{*}{ Haplotypes } & \multirow[t]{2}{*}{ No. of accessions } & \multirow[t]{2}{*}{ Percent $(\%)$} & \multicolumn{5}{|c|}{ Cultivated rice and ecological type and frequency (\%) } & \multicolumn{2}{|c|}{ Wild rice and frequency $(\%)$} \\
\hline & & & AUS & IND & TEJ & TRJ & ARO & O. nivara & O. rufipogon \\
\hline H1 & 14 & 34.1 & $2(50.0)^{\mathrm{c}}$ & 0 & $8(100)$ & $4(40.0)$ & 0 & 0 & 0 \\
\hline $\mathrm{H} 2$ & 8 & 19.5 & 0 & $5(62.5)$ & 0 & 0 & 0 & $3(60.0 \%)$ & 0 \\
\hline H3 & 5 & 12.2 & 0 & 0 & 0 & 0 & $5(100)$ & 0 & 0 \\
\hline $\mathrm{H} 4$ & 5 & 12.2 & 0 & $1(12.5)$ & 0 & $4(40.0)$ & 0 & 0 & 0 \\
\hline H5 & 3 & 7.3 & 0 & $2(25.0)$ & 0 & 0 & 0 & $1(20.0 \%)$ & 0 \\
\hline H6 & 1 & 2.4 & 0 & 0 & 0 & $1(10.0)$ & 0 & 0 & 0 \\
\hline $\mathrm{H} 7$ & 1 & 2.4 & 0 & 0 & 0 & 0 & 0 & $1(20.0 \%)$ & 0 \\
\hline H8 & 3 & 7.3 & $2(50.0)$ & 0 & 0 & $1(10.0)$ & 0 & 0 & 0 \\
\hline H9 & 1 & 2.4 & 0 & 0 & 0 & 0 & 0 & 0 & $1(100 \%)$ \\
\hline \multirow[t]{3}{*}{ Total } & 41 & 100.0 & 4 & 8 & 8 & 10 & 5 & 5 & 1 \\
\hline & f haplotypes & & 2 & 3 & 1 & 4 & 1 & 3 & 1 \\
\hline & of diversity ${ }^{d}$ & & 0.50 & 0.53 & 0.00 & 0.66 & 0.00 & 0.56 & 0.00 \\
\hline
\end{tabular}

${ }^{c}$ Number and frequency (in bracket) of rice accessions of each haplotype. ${ }^{\mathrm{d}}$ Diversity index was calculated as the frequency of haplotypes types in the accessions population following Fontaine's method (Fontaine et al., 2004). Diversity index $=\left(1-\sum_{i=1}^{\mathrm{n}} \mathrm{p}_{\mathrm{i}}^{2}\right)$ (where pi is the frequency of the haplotype $\mathrm{i}$ in a population).

Three amino acids, S321L, I441M, and H666R, of the Pi-d2 protein differed between $P i-d 2^{\text {Digu }}$ and $P i-d 2^{\mathrm{Np}}$. A single amino acid I at position 441 of the Pi-d2 protein determines resistant specificity, and $\mathrm{M}$ was found in the susceptible $p i-d 2$ allele in Nipponbare (Chen et al., 2006). The amino acids at this position in 9 haplotypes are summarized in Table 8. Amino acid $\mathrm{M}$ at position 441 was found in $\mathrm{H} 1$ and $\mathrm{H} 6$, suggesting that $\mathrm{H} 1$ and $\mathrm{H} 6$ are susceptible alleles; whereas, the remaining 7 haplotypes were identified as I at position 441 , suggesting they are resistance alleles. The functions of other amino acids among all Pi-d2 haplotypes are unknown. Interestingly, the amino acids of all the tested materials at position 666 were $\mathrm{R}$ (arginine), including Nipponbare. The I at position 441 was found in all wild rice accessions, suggesting that the resistance $P i-d 2$ allele was domesticated from wild rice relatives.

Table 8. Variation of the Pi-d2 protein in different accessions.

\begin{tabular}{|c|c|c|c|c|c|}
\hline \multirow[t]{2}{*}{ Haplotypes } & \multicolumn{5}{|c|}{ Variation locus ${ }^{\mathrm{e}}$} \\
\hline & 7 & 116 & 321 & 441 & 666 \\
\hline Digu & $\mathrm{R}$ & $\mathrm{F}$ & $\mathrm{S}$ & I & $\mathrm{H}$ \\
\hline Nipponbare & - & - & $\mathrm{L}$ & M & $\mathrm{R}$ \\
\hline $\mathrm{H} 1$ & - & - & $\mathrm{L}$ & M & $\mathrm{R}$ \\
\hline $\mathrm{H} 2$ & - & - & $\mathrm{L}$ & - & $\mathrm{R}$ \\
\hline H3 & - & - & - & - & $\mathrm{R}$ \\
\hline $\mathrm{H} 4$ & - & - & - & - & $\mathrm{R}$ \\
\hline H5 & - & $\mathrm{Y}$ & - & - & $\mathrm{R}$ \\
\hline H6 & - & - & $\mathrm{L}$ & M & $\mathrm{R}$ \\
\hline H7 & $\mathrm{H}$ & - & - & - & $\mathrm{R}$ \\
\hline H8 & - & - & - & - & $\mathrm{R}$ \\
\hline H9 & - & - & - & - & $\mathrm{R}$ \\
\hline
\end{tabular}

${ }^{e}$ Amino acid as that of $\mathrm{Pi}-\mathrm{d} 2$ in Digu. R, H, F, Y, S, L, I, and M = Arginine, Histidine, Phenylalanine, Tyrosine, Serine, Leucine, Isoleucine, and Methionine, respectively.

\section{Pathogenicity assays}

$\mathrm{H} 1$ and $\mathrm{H} 6$ contained M at the 441st amino acids of the Pi-d2 protein (Table 8), which were predicted to be the susceptible $p i-d 2$ alleles. From pathogenicity assays, rice accession 
Nipponbare was determined to be susceptible to the races IB1 and IEIK of M. oryzae, suggesting that Nipponbare has a susceptible $p i-d 2$ allele (Table 9). As shown in Table 9, reactions of the resistant haplotypes $\mathrm{H} 1, \mathrm{H} 3, \mathrm{H} 4$, and $\mathrm{H} 5$ did not correlate with the functional nucleotide of their respective structural variations.

\begin{tabular}{|c|c|c|c|}
\hline \multirow[t]{2}{*}{ Name of accession } & \multirow[t]{2}{*}{ Pi-d2 haplotype } & \multicolumn{2}{|c|}{ Disease reaction } \\
\hline & & IB1 $\left(\right.$ ARB146) ${ }^{\mathrm{Y}}$ & IEIK (TM2) \\
\hline IRGC30416(IR36) & H5 & R 0 & R 0 \\
\hline Nipponbare & H1 & S 4 & S 5 \\
\hline IRGC 1107 & $\mathrm{H} 1$ & S 5 & S 5 \\
\hline IRGC 418 & H1 & S 3 & S 5 \\
\hline IRGC 8244 & H1 & S 5 & S 5 \\
\hline IRGC 328 & H6 & S 5 & S 5 \\
\hline IRGC 8555 & $\mathrm{H} 1$ & S 5 & S 5 \\
\hline IRGC 6307 & $\mathrm{H} 8$ & R 1 & S 3 \\
\hline IRGC 27630 & $\mathrm{H} 1$ & S 3 & S 5 \\
\hline IRGC 32399 & $\mathrm{H} 1$ & R 0 & S 4 \\
\hline IRGC 38698 & $\mathrm{H} 1$ & S 5 & S 5 \\
\hline RA 4952 & $\mathrm{H} 3$ & R 0 & S 3 \\
\hline IRGC 43325 & $\mathrm{H} 4$ & S 4 & S 4 \\
\hline IRGC 43397 & $\mathrm{H} 8$ & R 0 & S 4 \\
\hline IRGC 43675 & $\mathrm{H} 4$ & S 5 & $\mathrm{R} 2$ \\
\hline IRGC 45975 & $\mathrm{H} 8$ & $\mathrm{R} 2$ & R 0 \\
\hline IRGC 50448 & $\mathrm{H} 4$ & $\mathrm{R} 2$ & R 1 \\
\hline IRGC 51250 & $\mathrm{H} 4$ & R 1 & R 0 \\
\hline IRGC 51300 & $\mathrm{H} 2$ & R 1 & S 3 \\
\hline IRGC 11010 & H1 & R 0 & S 3 \\
\hline IRGC 38994 & $\mathrm{H} 3$ & R 0 & S 5 \\
\hline IRGC 17757 & $\mathrm{H} 4$ & R 0 & S 3 \\
\hline IRGC 12793 & $\mathrm{H} 3$ & R 0 & S 3 \\
\hline IRGC 8191 & $\mathrm{H} 1$ & S 4 & R 0 \\
\hline IRGC 12883 & $\mathrm{H} 1$ & R 0 & S 4 \\
\hline
\end{tabular}

${ }^{\mathrm{x}}$ Only those accessions with seeds available from USDA were used for disease evaluation. Disease was evaluated using a 0-5 scale where 0-2 indicates resistance (R) and 3-5 indicates susceptibility (S), respectively. ${ }^{\mathrm{Y}}$ Field isolate ARB146 in 2009 from Arkansas described by Xing et al. (2013).

\section{Genetic structure and relationship of $P i-d 2$ in accessions}

To examine the population structure and relationships of $P i-d 2$ in 41 rice accessions, we constructed a phylogenetic tree based on the nucleic acid sequences of the $P i-d 2$ alleles (Figure 2). The rice accessions were divided into 2 clusters, 441I and 441M. The 441I cluster containing Digu, $O$. nivara, and O. rufipogon showed longer branches than that of the $441 \mathrm{M}$ cluster. However, the indica, japonica, O. nivara, and $O$. rufipogon groups were not separated, with IND being more dispersed and, thus, indicating higher diversity (Figure 2). The ARO was a specific cluster of $P i-d 2$. The tree for $P i-d 2$ showed the star-like branches in IND, O. nivara, and $O$. rufipogon, with long branches. These results suggest that the $P i-d 2$ resistance alleles may have originated from $O$. nivara.

\section{DISCUSSION}

Most cloned $R$ genes belong to the nucleotide binding site (NBS) and leucine-rich repeat (LRR) class of proteins and contain cytoplasmic proteins with a predicted leucine zip- 


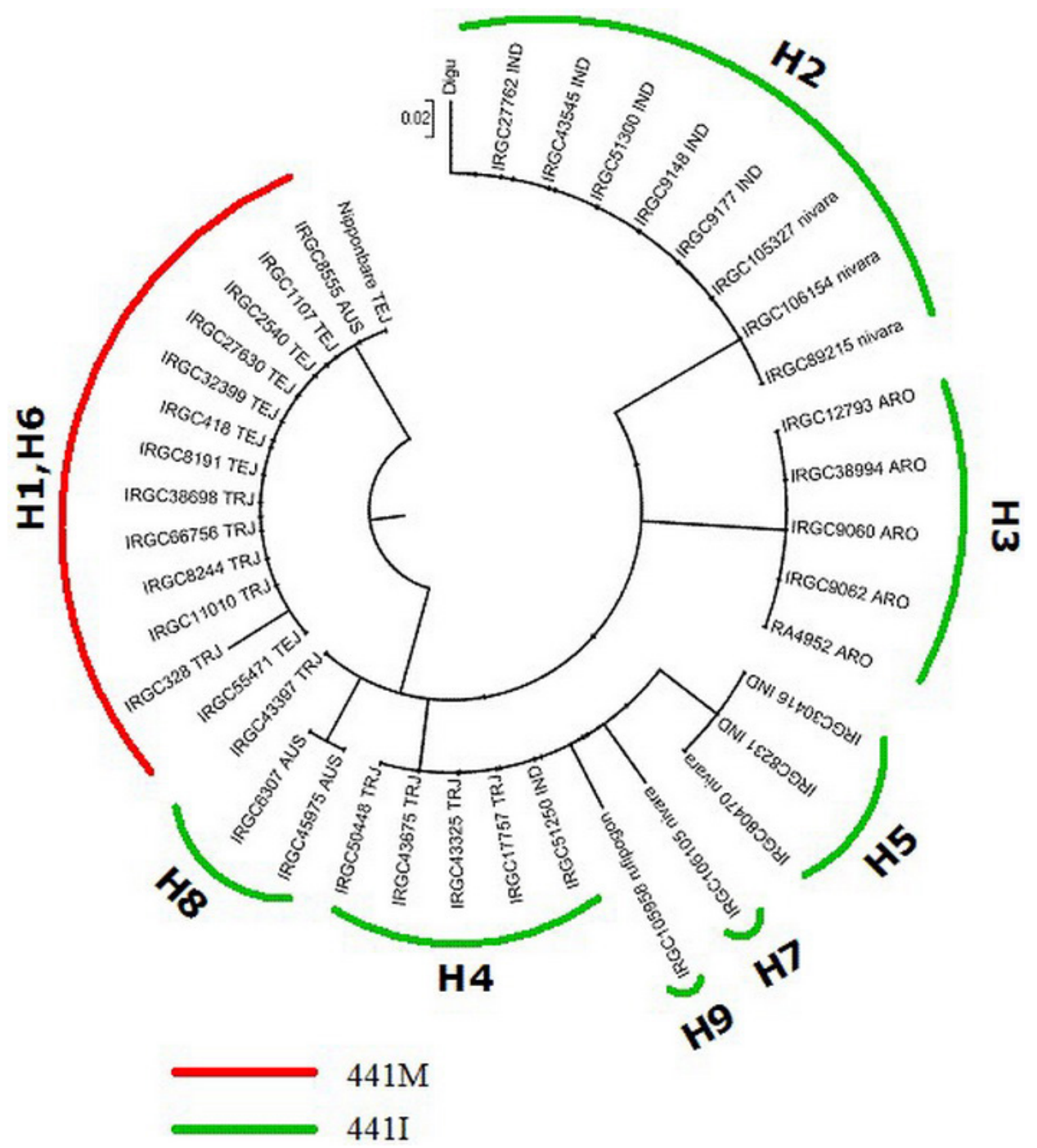

Figure 2. Phylogenetic tree constructed with the nucleotide sequences of $P i-d 2$ genes from 41 rice accessions ClustalW methods of DNASTAR V7.10. Digu carries $P i-d 2$ (Chen et al., 2006); 441M and 441I indicate the 441th amino acid were $\mathrm{M}$ and I of Pi-d2 proteins in rice accession, respectively; H1 to H9 indicate the haplotypes of Pi-d2.

per, NBS, and C-terminal LRR motifs. The $P i-d 2$ gene encodes a serine-threonine-RLK membrane-spanning protein. Because of its novel extracellular domain, $P i-d 2$ is a new class of rice $R$ genes. Similar to the NBS-type rice blast $R$ gene $P i$-ta, $P i-d 2$ is also a single-copy gene within the rice genome. $P i-d 2$ encodes a predicted protein of 825 amino acids with the domain characteristics of RLK, an extracellular domain, a transmembrane domain, and an intracellular kinase domain (Chen et al., 2006).

Studying the natural variation of $P i-d 2$ will help to understand the origin, evolution, and resistance stability of the rice $R$ gene. In the present study, we reported structural varia- 
tion of $P i-d 2$ in 41 cultivated and wild rice relatives. We found 13 SNPs, 11 of which are new, in the ORFs of Pi-d2 (Table 2). Some of the SNPs were specific to certain rice subgroups. A total of 9 haplotypes were identified based on 13 SNPs in 41 rice accessions. However, the resistant $P i-d 2$ allele in Digu and the 2 susceptible $p i-d 2$ alleles in cultivars LTH and TP309 were identical, except for 2 base changes at nucleotide positions 963 and 1323, respectively (Chen et al., 2006). These 2 base substitutions resulted in amino acid substitutions, S321L and $\mathrm{I} 441 \mathrm{M}$, in the susceptible $p i-d 2$ alleles. Translation of 9 haplotypes of $P i-d 2$ revealed 5 novel $\mathrm{Pi}-\mathrm{d} 2$ variants. Based on the functional amino acid, 5 new putative resistant haplotypes from cultivated and wild rice relatives were identified. Five variant amino acids of Pi-d2, including R7H, F116Y, S321L, I441M, and H666R, were found in 41 rice accessions when compared to Digu. Among them, the R7H and F116Y variant types were not reported in a previous study by Chen et al. (2006). The amino acids of all materials tested at position 666 were R (Arginine) and consistent with that of the Pi-d2 allele in Nipponbare; at the same position, H (Histidine) was present in Digu, suggesting that H666 is another functional amino acid for an unknown biological function that remains to be investigated.

An amino acid substitution in a critical functional domain often results in a conformational change of the protein that may impact the expected biological function. In the present study, we identified 9 amino acid variants of $\mathrm{Pi}-\mathrm{d} 2$ and 5 novel haplotypes in 41 rice accessions (Table 8). Three amino acid variants, R7H, F116Y, and H666R, of the Pi-d2 protein differed from those of $P i-d 2^{\text {Digu }}$. These amino acids are located in the hydrophobic region of the N-terminus, B-lectin of the extracellular domain, and serine-threonine kinase catalytic domain. These 3 amino acid variations may affect conformation of these functional domains, suggesting that there is the potential for instability of $\mathrm{Pi}-\mathrm{d} 2$-mediated rice blast resistance. In contrast, only one resistant haplotype, a single amino acid alanine at position 918 determining $P i$-ta resistance specificity, was found in 159 rice accessions of Oryza species, including AA genome species Oryza sativa, O. glaberrima, O. rufipogon, O. nivara, and O. barthii, and CC genome species $O$. officinalis (Wang et al., 2008). In this study, additional amino acid variants of Pi-d2 were found compared with that of Pi-ta, suggesting that confirmation of $\mathrm{Pi}-\mathrm{d} 2$ is influenced by more than just a single amino that was found in Pi-ta.

There was no correlation between the race-specific reaction and structural variation of Pi-d2, suggesting that other amino acids may influence reactions. This was not unexpected because the isolates/races IB1 (ARB14) and IE1k (TM2) of M. oryzae were used to determine the spectra of resistance mediated by the blast $R$ gene, Pi-ta (Xing et al., 2013). This analysis supported a previously known theory that there are lineage exclusions in field blast populations, where M. oryzae strains with similar $A V R$ genes have been asexually inherited. Nevertheless, these germplasm accessions may contain $P i$-ta that may mask their predicted reactions. Analysis of Pi-ta haplotypes in these germplasm accessions should help resolve this prediction. It is equally possible that other $R / A V R$ gene pairs in these germplasms can also mask expected reactions. Likewise, screening for the differential $M$. oryzae race of $P i$ $d 2$ will provide additional insight into the structural and functional relationships of the Pi-d2 haplotypes.

The functional amino acid at the 441 st position of Pi-d2 separated the cultivated accessions via 441M, and cultivated and wild rice relatives via 441I (Table 1; Figure 2). Despite not separating indica, japonica, O. nivara and O. rufipogon, the ARO subgroup was divided into a longer cluster, while TEJ was separated into a shorter cluster. Digu (with $P i-d 2$ ), IND, and nivara were grouped into one cluster, indicating that $\mathrm{Pi}-\mathrm{d} 2$ may have originated from $O$. 
nivara. IND was closely related to $O$. nivara, indicating a complex evolutionary history of $P i-d 2$ between IND and $O$. nivara. In fact, these rice accessions were divided into indica, japonica, $O$. nivara, and $O$. rufipogon groups based on the whole genome sequencing of rice accessions (Xu et al., 2011). These results suggest that uncoupling differential domestication processes had occurred in these germplasm accessions.

In contrast to most available genome sequencing datasets, we were able to extract $100 \%$ accurate $P i-d 2$ sequences (Xu et al., 2011) and use them to predict evolutionary histories and origins. The resulting knowledge will help to understand resistance stability and develop allelic specific DNA markers from portions of genes for use in marker-assisted selection (Jia, 2003).

\section{ACKNOWLEDGMENTS}

The authors gratefully thank Professor Wang Wen of the Kunming Institute of Zoology, Chinese Academy of Sciences for providing genome sequences of rice germplasm; Harold Bockelman of the USDA-ARS National Small Grains Collection, Aberdeen, Idaho, USA for providing germplasm accessions; Zaiquan Cheng of the Institute of Biotechnology and Genetic Resources, YAAS; Yiqing Guo of Yunnan Agricultural University for useful discussions; and Michael Lin, Tracy Bianco, and Ellen McWhirter of USDA-ARS Dale Bumpers National Rice Research Center for pathogenicity assays and proofreading, respectively. Research supported by the National Natural Science Foundation of China (\#31360331 and \#31160355) and Natural Science Fund of Yunnan of China (\#2010ZC173).

\section{REFERENCES}

Ballini E, Morel JB, Droc G, Price A, et al. (2008). A genome-wide meta-analysis of rice blast resistance genes and quantitative trait loci provides new insights into partial and complete resistance. Mol. Plant Microbe Interact. 21: 859-868.

Chen D, Chen X, Lei C, Ma B, et al. (2010a). Rice blast resistance of transgenic rice plants with Pi-d2 gene. Rice Sci. 17: 179-184.

Chen D, Chen X, Ma B, Wang Y, et al. (2010b). Genetic transformation of rice with $P i$ - $d 2$ gene enhances resistance to rice blast fungus Magnaporthe oryzae. Rice Sci. 17: 20-27.

Chen X, Li S, Xu J, Zhai W, et al. (2004). Identification of two blast resistance genes in a rice variety, Digu. J. Phytopathol. 152: 77-85.

Chen X, Shang J, Chen D, Lei C, et al. (2006). A B-lectin receptor kinase gene conferring rice blast resistance. Plant J. 46: 794-804.

Costanzo S and Jia Y (2010). Sequence variation at the rice blast resistance gene Pi-km locus: implications for the development of allele specific markers. Plant Sci. 178: 523-530.

Dai Y, Jia Y, Correll J, Wang X, et al. (2010). Diversification evolution of the avirulence gene AVR-Pital in field isolates of Magnaporthe oryzae. Fungal Genet. Biol. 47: 974-980.

Doron-Faigenboim A and Pupko T (2006). A combined empirical and mechanistic codon model. Mol. Biol. Evol. 24: 388-397.

Fjellstrom R, Conaway-Bormans C, McClung AM, Marchetti MA, et al. (2004). Development of DNA markers suitable for marker assisted selection of three Pi genes conferring resistance to multiple Pyricularia grisea pathotypes. Crop Sci. 44: 1790-1798.

Fontaine C, Lovett P, Sanou H, Maley J, et al. (2004). Genetic diversity of the shea tree (Vitellaria paradoxa C.F. Gaertn), detected by RAPD and chloroplast microsatellite markers. Heredity 93: 639-648.

Jia Y (2003). Marker assisted selection for the control of rice blast disease. Pesticide Outlook 14: 150-152.

Jia Y, Wang Z and Singh P (2002). Development of dominant rice blast resistance Pi-ta gene markers. Crop Sci. 42: 2145-2149.

Jia Y, Valent B and Lee FN (2003). Determination of host responses to Magnaporthe grisea on detached rice leaves using a spot inoculation method. Plant Dis. 87: 129-133. 
Jia Y, Redus M, Wang Z and Rutger JN (2004). Development of a SNLP marker from the Pi-ta blast resistance gene by tri-primer PCR. Euphytica 138: 97-105.

Hao Z, Wang J, Wang L and Tao R (2009). Influences of the disease resistance conferred by the individual transgenes, $P i-d 2, P i-d 3$ and $X a 21$, on the transgenic rice plants in yield and grain quality. African J. Biotechnol. 8: 4845-4848.

Hittalmani S, Parco A, Mew TW, Zeigler RS, et al. (2000). Fine mapping and DNA marker-assisted pyramiding of the three major genes for blast resistance in rice. Theor. Appl. Genet. 100: 1121-1128.

Lei J, You N, Huang L, Lei S, et al. (1998) Breeding and utilization of indica rice CMS line Fuyi A. Hybrid Rice 13: 8-13

Lei J, You N, Hu A, Lei S, et al. (2004). Research and utilization of resistance to blast in indica rice CMS line Fuyi A. $J$. Fujian Agric. Forestry Univ. (Natural Science Edition) 33: 141-147.

Lee S, Costanzo S, Jia Y, Olsen K, et al. (2009). Evolutionary dynamics of the genomic region around the blast resistance gene Pi-ta in AA genome Oryza species. Genetics 183: 1315-1325.

Lee S, Jia Y, Jia M, Gealy D, et al. (2011). Molecular evolution of the rice blast resistance gene Pi-ta in invasive weedy rice in the USA. PLoS One 6: e26260.

Okuyama Y, Kanzaki H, Abe A, Yoshida K, et al. (2011). A multi-faceted genomics approach allows the isolation of rice Pia blast resistance gene consisting of two adjacent NBS-LRR protein genes. Plant J. 66: 467-479.

Rozas J, Sánchez-DelBarrio JC, Messeguer X and Rozas R (2003) DnaSP, DNA polymorphism analyses by the coalescent and other methods. Bioinformatics 19: 2496-2497.

Sharma TR, Rai AK, Gupta SK, Vijayan J, et al. (2012). Rice blast management through host-plant resistance: retrospect and prospects. Agric. Res. 1: 37-52.

Silue D, Notteghem J and Tharreau D (1992). Evidence for a gene-for-gene relationship in the Oryza sativa-Magnaporthe grisea pathosystem. Phytopathol. 82: 577-580.

Swanson W, Nielsen R and Yang Q (2003). Pervasive adaptive evolution in mammalian fertilization proteins. Mol. Biol. Evol. 20: 18-20.

Tajima F (1989). Statistical method for testing the neutral mutation hypothesis by DNA polymorphism. Genetics 123: 585-595.

Tamura K, Dudley J, Nei M and Kumar S (2007). MEGA4: molecular evolutionary genetics analysis (MEGA) software version 4.0. Mol. Biol. Evol. 24: 1596-1599.

Wang X, Jia Y, Shu Q and Wu D (2008). Haplotype diversity at the Pi-ta locus in cultivated rice and its wild relatives. Phytopathology 98: 1305-1311.

Wasano N, Ohgushi A and Ohba M (2003). Mannose-specific lectin activity of parasporal proteins from a lepidopteraspecific Bacillus thuringiensis strain. Curr. Microbiol. 46: 43-46.

Xing J, Jia Y, Correll J, Lee F, et al. (2013). Analysis of genetic and molecular identity among field isolates of rice blast fungus using an international differential system, Rep-PCR and DNA sequencing. Plant Dis. 97: 491-495.

Xu X, Liu X, Ge S, Jensen J, et al. (2011). Resequencing 50 accessions of cultivated and wild rice yields markers for identifying agronomically important genes. Nat. Biotechnol. 30: 105-111.

Yang Z, Nielsen R, Goldman N and Pedersen A (2000). Codon-substitution models for heterogeneous selection pressure at amino acid sites. Genetics 155: 431-449.

Zhou E, Jia Y, Singh P, Correll J, et al. (2007). Instability of the Magnaporthe oryzae avirulence gene AVR-Pita alters virulence. Fungal Genet. Biol. 44: 1024-1034. 